\title{
Optimal path selection algorithm for mobile beacons in sensor network under non-dense distribution
}

https://doi.org/10.1515/phys-2018-0127

Received October 3, 2018; accepted November14, 2018

Abstract: When the traditional anchor aided location algorithm is used to select the mobile beacon path in the sensor network, there is no analysis of the energy imbalance of nodes in non-dense conditions, the optimal network node cannot be selected, and the selection error of the optimal path of the beacon is larger. A path selection algorithm for mobile beacons in a sensor network under non-dense distribution is proposed. Using the mobile beacon based wireless sensor network location algorithm, the weighted centroid algorithm and the extended Kalman filter (EKF) are used to obtain the accurate location results of the unknown nodes around the mobile beacon in the sensor network under non-dense distribution condition. The optimal node energy partition of the unknown node is obtained by the chaotic differential evolution method, and the optimal location of the optimal energy node in the wireless sensor network is calculated using the dynamic escape particle swarm optimization method, and the optimal beacon path is extracted. The experimental results show that the proposed algorithm can enhance the clustering performance of the optimal node in the wireless sensor network and has a better performance of dynamic node selection in wireless sensor network, and the convergence speed is faster and the operation time is shorter.

Keywords: Non-dense distribution, sensor, mobile beacon in network, path optimization, dynamic escape particle swarm optimization, chaotic differential evolution

PACS: 89.20.Ff, 07.05.Kf, 07.07.Mp

\footnotetext{
*Corresponding Author: Guoqing Yu: School of Information Science and Engineering, Hebei University of Science and Technology, Shijiazhuang, 050018, China,

E-mail: hbkdygq@163.com

Hongtao Ma: School of Information Science and Engineering, Hebei University of Science and Technology, Shijiazhuang, 050018, China, E-mail: dzxmht@163.com

Deden Witarsyah: School of Industrial Engineering, Telkom University, 40257, Bandung, West Java, Indonesia, E-mail:
}

\section{Introduction}

With the increasing application of wireless sensor networks, their performance requirements have gradually improved. In wireless sensor networks, the energy of nodes is limited, and this is the key factor that interferes with the efficiency of communication. Under the condition of non-dense node distribution, the rational use of node resources to optimize the structure of sensor networks is the key method to expand the application of wireless sensor networks, and it has become a hot issue in this field. For example, Huang et al. [1] proposed the use of a historical beacon and RSS to obtain three constraints and introduce a range-free location algorithm with low communication cost. When receiving the signal strength, the algorithm was disturbed by noise, resulting in inaccurate reception results. Halder \& Ghosal [2] proposed a mobile anchor aided localization algorithm to reduce implementation costs by using a limited number of mobile anchors. The algorithm did not fully analyze the problem of node energy imbalance under non-dense condition, and cannot select the best network nodes. Alomari et al. [3] introduced a new static path planning model. This model ensured that all nodes in the sensor network could receive location information. Compared with a similar static model, the new static path planning model estimated its own location, with high positioning accuracy, but had low communication efficiency and large defects. Sun et al. [4] proposed a beacon deployment strategy to ensure location in wireless sensor networks. However, due to the constraints of the network, the strategy had some limitations. Singh \& Khilar [5] proposed an analytical geometric distance free location scheme based on mobile beacon points in wireless sensor networks, but the analysis of the scheme was complex and inefficient.

In view of the above problems, this paper proposes an algorithm for mobile beacon path selection in wireless sen-

dedenw@telkomuniversity.ac.id 
sor networks under non-dense distribution. Firstly, a localization method based on mobile beacon is designed to accurately locate the mobile beacon path in wireless sensor networks under non-dense distribution. Then the node selection optimization algorithm based on chaotic differential evolution and dynamic escape particle swarm optimization is used to select the optimal path of mobile beacon in the sensor network under non-dense distribution.

\section{Methods}

\subsection{Node localization algorithm in wireless sensor network based on mobile beacon}

\subsubsection{The principle of positioning}

\section{Mobile model of beacon}

In the process of robot localization, the robot can traverse the whole area according to the artificial track. However, the key difference is that the mobile beacon in the sensor network under non-dense distribution has little or only small control [6]. At present, the mobile models for non-dense distributed sensor networks include random mobility model and S-type. The random mobile model has a small requirement for the hardware configuration of the beacon, but it is difficult to cover the whole non dense sensing area, resulting in low location coverage [7]. Although S-type and other regular models can traverse the sensing area according to the pre-trajectory, they require high hardware configuration of beacons [8]. In this paper, the Gauss Markov model is used for mobile beacons. The model does not require significant hardware configuration of beacon and can cover the whole non-dense sensing area. The Gaussian Markov model is defined as follows:

$$
\begin{aligned}
& v_{k}=\alpha v_{k-1}+(1-\alpha) v_{\text {mean }}+\gamma_{v} \sqrt{1-\alpha^{2}} \\
& d_{k}=\alpha v_{k-1}+(1-\alpha) d_{\text {mean }}+\gamma_{d} \sqrt{1-\alpha^{2}}
\end{aligned}
$$

Where, $v_{k}$ and $d_{k}$ represent the speed and direction of the time $k . v_{\text {mean }}$ is the average speed, is the average direction of motion, $\gamma_{v}$ and $\gamma_{d}$ represent Gauss random variables, and $\alpha$ is the adjustment parameter of randomness, then the location of the non-dense sensor network is as follows:

$$
\begin{aligned}
& x_{k}=x_{k-1}+v_{k-1} \times \cos \left(d_{k-1}\right) \\
& y_{k}=y_{k-1}+v_{k-1} \times \cos \left(d_{k-1}\right)
\end{aligned}
$$

Where, $x_{k}$ and $y_{k}$ represent beacon movement to the horizontal and vertical positions of $k$ th intervals respectively.

Distance measuring method

This paper calculates the distance between unknown node and mobile beacon by using the received signal strength indication (RSSI) value and propagation path loss empirical model [9]. In the actual application environment, the log-normal distribution model of random interference is usually introduced as the path loss model of wireless sensor networks due to factors such as multipath, diffraction and obstacles.

$$
P L(d)=P L\left(d_{0}\right)+10 n \lg \left(\frac{d}{d_{0}}\right)+X_{\sigma}
$$

Where, $d_{0}=1 \mathrm{~m}$ is the reference distance; $P L\left(d_{0}\right)$ is the path loss after passing the distance of $d_{0} ; d$ is the real distance; $P L(d)$ is the path loss after the beacon signal reaches the unknown node; $X_{\sigma}$ is the shielding factor of the average value of zero with the unit $d B ; n$ is the path loss index, and its value depends on the surrounding environment and the type of the building.

Weighted centroid algorithm model

The weighted centroid algorithm uses the former $\mathrm{m}$ virtual beacons with the largest RSSI value received by each unknown node as the location reference information, in order to ensure the positioning accuracy [10]. There is not a unified standard for determining weighted centroid values, but the choice of weights must ensure that the beacon nodes which are closer to the unknown nodes have the greater weight value [11]. This paper proposes that the weight value corresponds to the reciprocal of distance: $w_{1}: w_{2}: \cdots: w_{m}=1 / d_{1}: d_{2}: \cdots: 1 / d_{m}$. The weight selection is simple and feasible, and it fully meets the basic requirements of weight selection. Such an unknown node can be expressed as:

$$
X_{\text {est }}=\sum_{i=1}^{m}\left(X_{i} \times\left(w_{i} / \sum_{j=1}^{m} w_{j}\right)\right)
$$

Where, $X_{i}$ are the received coordinate $\left(x_{i}, y_{i}\right)$ of the ith virtual beacon, $X_{\text {est }}$ is the initial estimated coordinate of unknown node, and $w_{j}$ is the unknown node.

Establishment of the extended Calman filtering model [12]

Simulation of state equation is:

$$
X_{k}=A \times X_{k-1}+w_{k}
$$

$X_{k}=\left(x_{k}, y_{k}\right)^{T}$ represents the coordinate vector of unknown nodes after filtering of the $k-1$ beacon. $w_{k}$ is the measurement process noise, and $A$ is the second order unit matrix. 
The simulation of observation equation is:

The distance between unknown nodes and beacons is used as the observation quantity $\left(Z_{k}\right)$ of extended Kalman filter tracking distance. The observation equation is as follows:

$$
Z_{k}=f\left(X_{k}\right)+v_{k}=H \times X_{k}+v_{k}
$$

The observation equation is linearized [13]:

$$
Z_{k}=\left[\begin{array}{ll}
\frac{\partial d n}{\partial x} & \frac{\partial d n}{\partial y}
\end{array}\right] \times X_{k}+v_{k}
$$

Where, $d_{k}=\sqrt{\left(x b_{k}-x_{k}\right)^{2}+\left(y b_{k}-y_{k}\right)^{2}} . \quad d_{k}$ is the distance between the unknown node and the $k$ virtual beacon, $\left(x b_{k}, y b_{k}\right)$ is the coordinate of the $k$ beacon of the unknown node, and $v_{k}$ is the observation noise, the Gauss distribution random number with zero mean value, and its standard deviation is $\sigma_{1}$. Of which $H$ is the Jacobi matrix of $f\left(X_{k}\right)$.

The EKF calculation process is as follows:

The current state is predicted using the previous state of the system:

$$
X(k \mid k-1)=A X(k-1 \mid k-1)
$$

The covariance of the current state of the system is updated:

$$
P(k \mid k-1)=A P(k-1 \mid k-1) A^{T}+W Q W^{T}
$$

The extended Calman gain is computed as:

$$
K g(k)=P(k \mid k-1) H^{T}\left(H P(k \mid k-1) H^{T}+V R V^{T}\right)^{-1}
$$

Combining the predicted and observed values, the optimal estimated value of the present state is estimated:

$$
X(k \mid k)=X(k \mid k-1)+K g(k)(Z(k)-H X(k \mid k-1))
$$

The covariance of the present optimal estimated value is updated:

$$
P(k \mid k)=(1-K g(k) H) P(k \mid k-1)
$$

Making cyclic iteration, and then return step (1).

Where, $Q$ is the covariance matrix of process noise: $Q=0 ; R$ is the covariance matrix of observation noise: $R=\sigma_{1}^{2} ; \sigma_{1}$ is the standard deviation of Gaussian distribution random number $v_{k}$. W is the Jacobian matrix of the state equation and is the Jacobian matrix of the observation equation.

\subsubsection{Design of the algorithm}

In this paper, a mobile beacon is first used to allow the Gauss Markov model to traverse the sensing area, and to publish the location information periodically. The unknown node processes the location information [14]. Then, the initial coordinates of the unknown nodes are calculated by the weighted centroid algorithm. Finally, the EKF iteration cycle is used to converge the initial position coordinates to the true coordinates of the unknown nodes [15]. The concrete steps of the algorithm are as follows:

The mobile beacon traverses the entire sensing area.

The Gauss Markov model is used by the Mobile beacon to periodically broadcast the location and RSSI packets during the mobile process.

The unknown node processes the received virtual beacon [16].

a. the unknown node receives and records the virtual beacon information $\left[\left(x b_{i}, y b_{i}\right) ; R S S I\right] ; i=1,2, \ldots, m$ after receiving the information. $m>=3$.

b. the recorded information is sorted [17]. According to the ranking of RSSI values from large to small, if the RSSI value is equal, the arrangement is not sequential.

c. the set of distance observations from unknown nodes to beacon locations are recorded: $D=\left\{d_{1}, d_{2}, \ldots, d_{m}\right\}, i=$ $1,2, \ldots, m . d_{i}$ corresponds to the received sequence $R S S I_{i}$ and $d_{i}$ from the big to the small, and when the distance value is the same, the arrangement is not sequential.

(3) The weighted centroid method is used to calculate the approximate location $X_{e s t}=\sum_{i=1}^{m}\left(X_{i} \times\left(w_{i} / \sum_{j=1}^{m} w_{j}\right)\right)$ of unknown nodes.

(4) The approximate location of unknown nodes provided by step (3) is used to determine the initialization state of EKF. The EKF loop iteration is used to precisely locate every unknown node.

\subsection{Path selection algorithm for mobile beacon in wireless sensor networks under non-dense distribution}

According to the precise location of each unknown node obtained in the upper section, the optimal path of the mobile beacon is obtained using the optimal path selection algorithm of mobile beacon in the sensor network under non- dense distribution [18], and the detailed process is:

Chaos differential evolution method is used to obtain the optimal node energy partition of unknown nodes.

In order to describe the diversity of the direct groups of different sub-regions in the sensor networks under non- 
dense distribution, the variance of the fitness value of the data group and the diversity factor $m f$ are set, and then there are:

$$
m f=\frac{1}{N P} \sum_{i=1}^{N P}\left(f\left(x_{i}\right)-\bar{f}(x)\right)^{2}
$$

Where, $N P$ represents the size of the sensor networks under non-dense distribution, $f\left(x_{i}\right)$ is the fitness value of $i$ th chromosome in similar genetic algorithm, and $\bar{f}(x)$ is the average fitness value.

In the iterative process, in order to avoid the algorithm entering the local optimal value too early, it is necessary to integrate the perturbation theory of chaotic time series to improve the algorithm performance. According to the chaos theory, the optimal search of unknown nodes is carried out through the Logistic chaotic time series shown in Eq. (16) [19].

$$
x_{n+1}=\mu x_{n}\left(1-x_{n}\right)
$$

Where, $n=1,2,3, \ldots, x \in[0,1] \mu \in[0,4] . x$ is to simulate sequence elements, $n$ is an iterative time step, $\mu$ is an adjustable parameter. Through numerical iteration, it is found that

If $0 \leq \mu \leq 1$, the iteration system has only $x=0$ stable 1 - periodic point.

If $0 \leq \mu \leq 3$, the iterative system has an unstable 1periodic point $x=0$, and a stable 1-periodic point $x=1-$ $1 / \mu$.

If $3 \leq \mu \leq 3.449$, there are two unstable 1-periodic points and $x=1-1 / u$.

$$
\begin{aligned}
& x=\frac{1}{2}(1+\mu \sqrt{(\mu+1)(\mu-3)}) \\
& x=\frac{1}{2 \mu}(1+\mu \sqrt{(\mu+1)(\mu-3)})
\end{aligned}
$$

Eq. (17) and (18) are two stable 2-periodic points of the system: when $3.449 \leq \mu \leq 3.544$, the periodic point becomes unstable, and four stable 4-periodic points appear in this case.

When parameter continues to increase, $\mu>3.544$, the 4-periodic point and eight stable 8-periodic solutions. If it continues to iterate, it will find that the 8- periodic solutions fluctuate, and there are 16 stable periodic solutions, so go on, then.

Based on the characteristics of chaotic variables, the optimal search of the unknown nodes in the sensor network under non-dense distribution is carried out [20]. The chaos disturbance is integrated into the unknown node partition process, and the optimization of the unknown node partition of the sensor network is completed under the non- dense condition, and the best energy of the unknown node is obtained [21].

The optimal location of nodes in the wireless sensor network is found.

According to the above process, the optimal energy node of unknown nodes in the sensor network is obtained under non-dense distribution. By using the dynamic escape particle swarm optimization method, the optimal position of the optimal energy node in wireless sensor networks is calculated, and the network node coverage optimization is realized [22]. The dimension of wireless sensor network space is set to $k$, the number of particles in the dynamic escape particle swarm is $n, Y_{k}=$ $\left(y_{j 1}, y_{j 2}, \ldots, y_{j k}\right)$ represents the space position of the $j$ th particle. The speed of particle motion is represented by $W_{j}=\left(w_{j 1}, w_{j 2}, \ldots, w_{j k}\right)$. The optimal space position in the process of particle motion is represented by $Q_{j}=$ $\left(q_{j 1}, q_{j 2}, \ldots, q_{j k}\right)$, and the best space position of all particles in the global search process can be calculated by the following formula.

$$
\begin{aligned}
w_{j k}^{l+1}= & x(l) \times w_{j k}^{l} \\
& +d_{1} s_{1 k}\left(\text { pbest }_{j k}^{l}-y_{j k}^{l}\right) d_{2} s_{2 k}\left(\text { pbest }_{j k}^{l}-y_{j k}^{l}\right) \\
y_{j k}^{l+1}= & y_{j k}^{l}+w_{j k}^{l}
\end{aligned}
$$

Where, $d_{1}$ is the weight coefficient of the running speed of the dynamic escape particle, and the $d_{2}$ is the weight coefficient of the running speed of all the particles in the wireless sensor network, $s_{1 k}$ and $s_{2 k}$ are the random numbers in the range of $0 \sim 1$, and $y_{j k}^{l}$ is the th dimensional space position parameter of the dynamic escape particle $j$ during the first iteration process, and $w_{j k}^{l}$ is the running speed of its corresponding escaping particles. pbest ${ }_{j k}^{l}$ is the spatial escape position of particle $j$ in the $k$ th dimension, and $x$ is the inertia weight coefficient. Through the operation, it can be obtained

$$
x(l)=0.9 w_{j k}^{l+1}-0.5 \times l / \max -\text { step }
$$

Where, $l$ is the number of iterations for network node coverage by dynamic escape particle swarm optimization, and max -step is the maximum number of iterations.

The following formula can be used to calculate the spatial location parameter mbest of the coverage area in wireless sensor network under non- dense distribution.

$$
\begin{aligned}
\text { mbest } & =x(l) \sum_{j=1}^{N} q_{j} / N \\
& =x(l)\left(\sum_{j=1}^{N} q_{j 1} / N, \sum_{j=1}^{N} q_{j 2} / N, \ldots, \sum_{j=1}^{N} q_{j k} / N\right)
\end{aligned}
$$


Where, 1 is the spatial position parameter of dynamic escape particle $j$ in th dimension. $N$ is the number of nodes.

The detailed process of mobile beacon's path selection under non- dense distribution is as follows:

Initialization. Firstly, the initialization of node energy control parameters, including the maximum number of iterations, dimensions, iterations and disturbance range, is carried out. Then the node area is initialized, and a random matrix is randomly generated as the initial population, and then an initial membership matrix is generated to select the initial optimal individual and the global optimal individual.

An evolutionary computation is carried out according to the differential evolution algorithm [23].

The threshold $\zeta$ is set, the diversity factor is calculated, and $m f$ and $\zeta$ are made comparison analysis. If $m p>$ $\zeta$, then go to step (4), if $m f>\zeta$, then go to step (5);

(4) The chaotic sequence is integrated into the partition to produce a random matrix $z$, which is $c \times D$, and each component is between $(0,1)$, and $N P$ chaotic sequence variables are obtained according to the Logistic chaotic mapping.

$$
x_{n+1}=4 x_{n}\left(1-x_{n}\right), n=1,2, \ldots, N P
$$

The chaotic components that integrate into the individual perturbation variables are:

$$
\Delta x_{i}=a+(b-a) x_{n}, n=1,2, \ldots, N P
$$

The perturbed variables that are loaded into the population are:

$$
x_{n, G}=x_{n, G}+\Delta x_{i}
$$

The distance from each data to the clustering center is calculated, to adjust $U$. The population is updated through the new $U$, and the individual with the smallest fitness is the optimal individual of the present generation.

The optimized coverage of the wireless sensor network nodes is carried out by the dynamic escape particle swarm optimization (DDE-PSO) algorithm to calculate the optimal location of wireless sensor network node mbest.

If $G=$ mbest $G_{\max }$, the iteration is stopped and the global optimal individual and the optimal fitness value are the output. If it is not, then $G=G+1$, and make iteration with the step (2).

The above analysis process is based on the uniform traversal characteristics of chaotic sequences and the efficient global search ability of differential evolution algorithm. Chaotic perturbation is incorporated into the node energy partition process to get the best energy node. Dynamic escape particle swarm optimization is used to calculate the optimal position of the optimal energy node in wireless sensor networks, which is the optimal beacon path.

\section{Results}

\subsection{Experimental setting}

In order to improve the performance of the mobile beacon path optimal selection algorithm in the sensor network under non-dense distribution, the related simulation experiments are carried out. The simulation conditions are as follows: setting up the target area of wireless sensor network is the flat area $100 \mathrm{~m} \times 100 \mathrm{~m}$. The radio transmitting parameters of the mobile beacon node refer to the experimental data measured by MICA2mote, in Motorola Florida R\&D Center, the attenuation factor in formula (5) is $n=2.38$, and the standard deviation of the Gauss distribution random variable is $\sigma_{d B}=7.98 \mathrm{~dB}$. The communication radius of each unknown node is $r=10 \mathrm{~m}$ (the greater the communication radius is, the more location information can be obtained, but at the same time, the higher the cost of the nodes is, so the $10 \mathrm{~m}$ communication radius that the current sensor nodes can basically reach is chosen). The noise $\gamma_{v}$ and $\gamma_{d}$ in Eq. (1) and Eq. (2) are all subject to Gauss random distribution, with a mean value of 0 and a variance of 1 . The noise value of the model increases a certain degree of freedom, making it more close to the beacon movement affected by various environmental parameters. In order to make the beacon to move beyond the boundary of the sensing area, the average speed $v_{\text {mean }}$ is not larger and set as $2 \mathrm{~m} / \mathrm{s}$, the average motion direction $d_{\text {mean }}$ is set to $90^{\circ}$, that is, the direct direction. When the beacon approaches the boundary, $d_{\text {mean }}$ should change the beacon into the sensing area with the change [24]. The randomness adjustment parameter $\alpha=0.6$ is set, $\alpha$ is a number between 0 and 1 , the larger the value of $\alpha$ is, the greater the model's dependence on the previous state is and the enhancement of the memory is [25-31].

50 sensor nodes are randomly distributed in the plane area, and the starting point of the beacon is $(50,50)$. According to the Gauss Markov model, the beacon moves $2500 \mathrm{~s}$, so that the whole sensing area is basically covered.

\subsection{Analysis results}

If the moving time of a certain beacon is $3000 \mathrm{~s}$, the average speed is $2 \mathrm{~m} / \mathrm{s}$, the interval of the broadcast information is $1 \mathrm{~s}$, and the number of iterations is 50 , the number of 
virtual beacons received is changed, the number of virtual beacons varies from 3 to 27 (the number of virtual beacons received by each unknown node is different, the number of virtual beacons selected is based on the RSSI value). The location of ten unknown nodes is simulated and the average value is taken. The anchor assistant location algorithm, the free location algorithm and the proposed algorithm are compared, and the corresponding location error change curves of mean square root are shown in Figure 1.

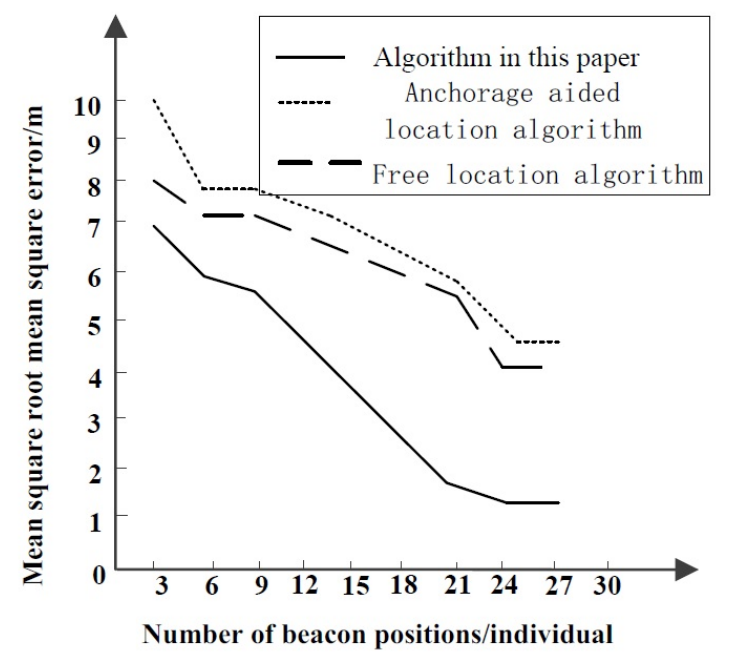

Figure 1: Curves of virtual beacon number and mean square root error

If the number of virtual beacons received by an unknown node is 21 , the moving time of the mobile beacon is $3000 \mathrm{~s}$, the interval of the mobile beacon broadcast information is $1 \mathrm{~s}$ and the number of iterations is 50 , the motion speed of the beacon is changed, the ten unknown nodes are located and simulated, and the average value is taken, then the average root mean square error curve of the algorithm proposed in this paper is as shown in Figure 2.

If the number of virtual beacons received by an unknown node is 21, the moving time of the mobile beacon is $3000 \mathrm{~s}$, the interval of the mobile beacon broadcast information is $1 \mathrm{~s}$ and the number of iterations is 50 times, the motion speed of the beacon is changed, and the unknown nodes are located and simulated, then the change of coverage rate by using the algorithm proposed in this paper is as shown in Figure 3.

If the number of virtual beacons received by unknown nodes is 21 , the moving speed of the mobile beacon is $2 \mathrm{~m} / \mathrm{s}$, the interval of the mobile beacon broadcast information is $1 \mathrm{~s}$ and the number of iterations is 50 times, the moving time of the beacon in the sensing area is changed,

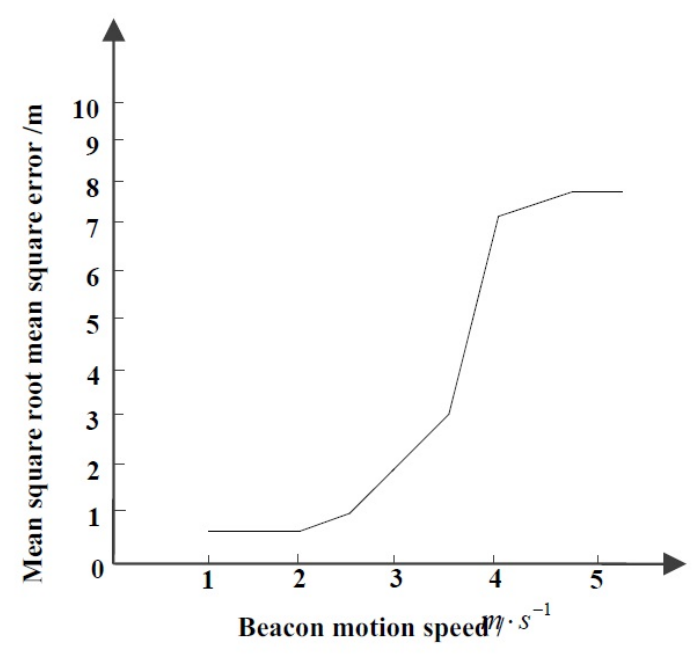

Figure 2: Graph of motion speed and mean square root error of algorithm beacon in this paper

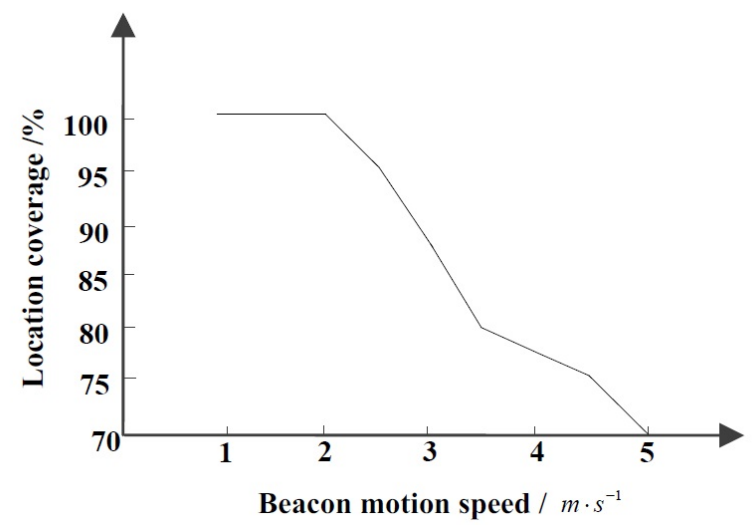

Figure 3: Graph of beacon velocity and location coverage of algorithm in this paper

and the change curve of the localization rate by using the proposed algorithm is shown in Figure 4.

If the number of virtual beacons received by unknown nodes is 21 , the running time of the mobile beacon is $3000 \mathrm{~s}$, the speed is $2 \mathrm{~m} / \mathrm{s}$, the interval of broadcast information is $1 \mathrm{~s}$, the number of EKF iterations is changed, and the average value mean square error curve of the ten unknown nodes is simulated and the average root mean square error curve of the proposed algorithm is shown as Figure 5.

After locating the mobile beacon in the sensor network under non-dense distribution, the anchor aided location algorithm, the free location algorithm and the proposed algorithm are used to optimize the path selection of the mobile beacon in the wireless sensor network under the nondense distribution. The rectangular frame is used to describe the target area of the wireless sensor network node, 


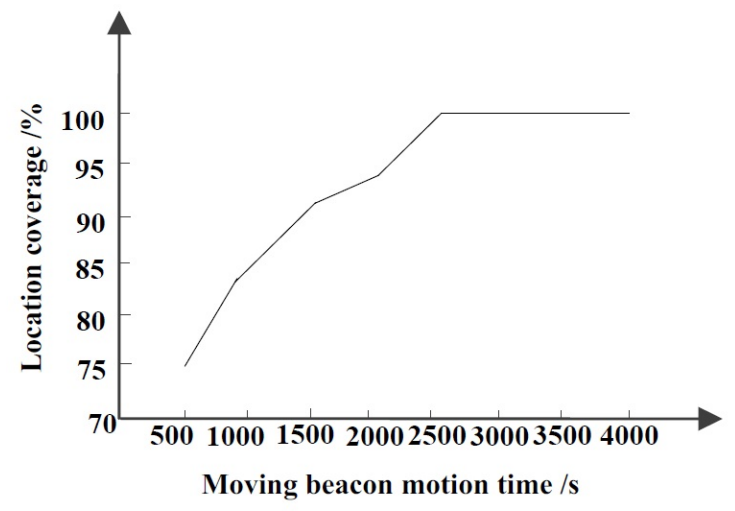

Figure 4: Graph of beacon mobile time and location coverage in this algorithm

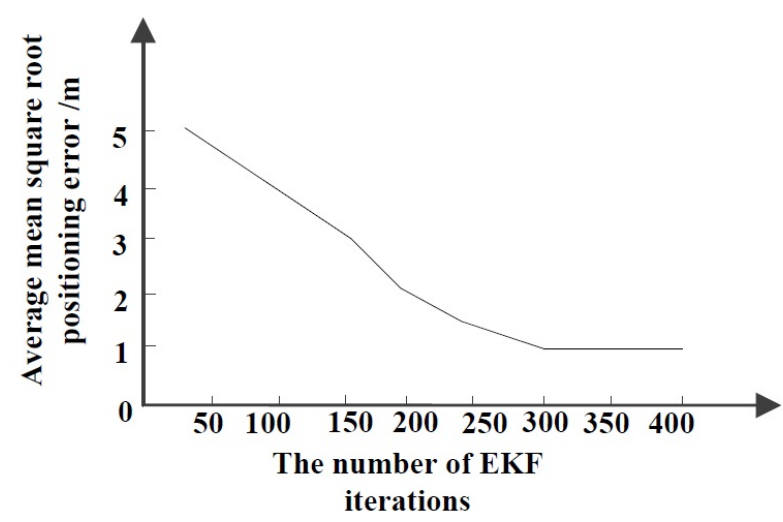

Figure 5: Graph of EKF iterations and mean root mean square error of the algorithm

the prototype is used to describe the sensor radius, and the solid point is used to describe the spatial location of the node. The optimization results are described in Figures 6 and 7 , respectively.

100 separate dynamic nodes selection simulation experiments for wireless sensor networks are conducted respectively. The proposed algorithm, the anchor aided location algorithm and the free location algorithm are simulated, and the average optimum fitness, the average operation time and the average iteration number of the three algorithms are obtained. The detailed results are described in Table 1.

\section{Discussion}

As can be seen from Figure 1, as the number of beacon nodes increases, the average mean square root positioning error of the three algorithms decreases, but the degree

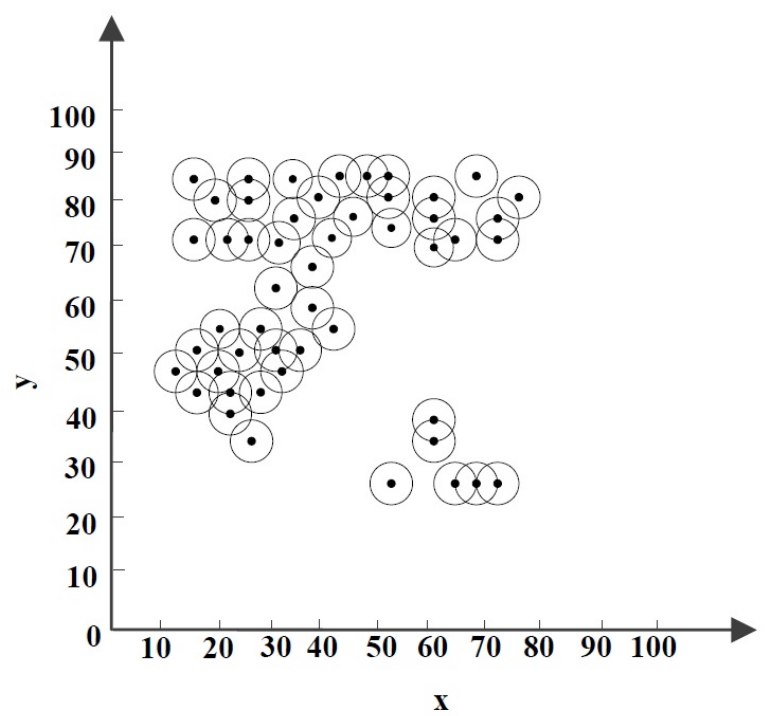

Figure 6: Node selection result of wireless sensor network based on anchor aided localization algorithm

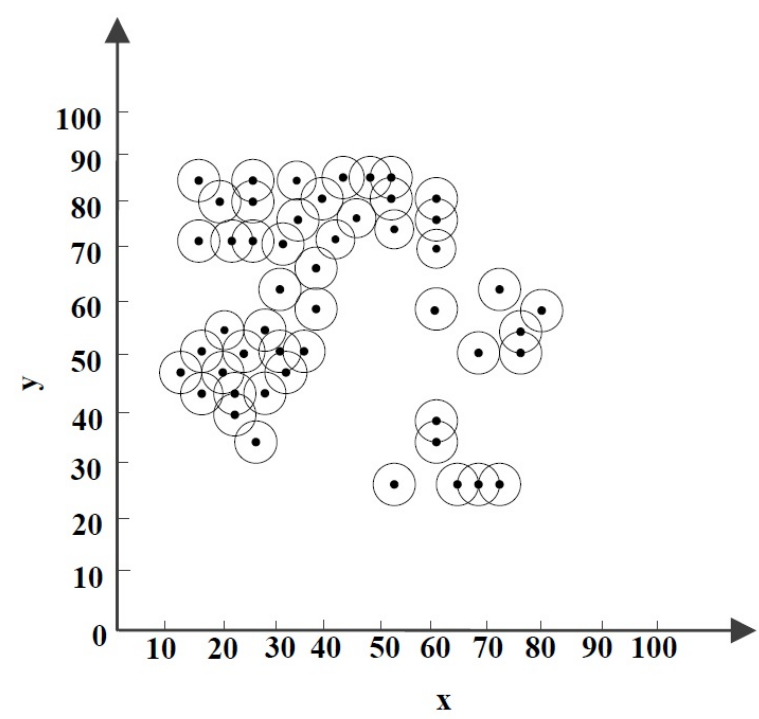

Figure 7: Node selection result of wireless sensor network based on free localization algorithm

of reduction decreases slowly. When the number of virtual beacons received by an unknown node is 3 , the mean square root positioning errors of the three algorithms are $9.5 \mathrm{~m}, 8 \mathrm{~m}$ and $6.7 \mathrm{~m}$ respectively. When the number of virtual beacons is 27 , the corresponding average root mean square error is $4.6 \mathrm{~m}, 4.1 \mathrm{~m}$, and $1.7 \mathrm{~m}$. The average root mean square error of the proposed algorithm is stable when the virtual beacon reaches over 21 , indicating that the proposed algorithm is obviously better optimized than two other algorithms. 
Table 1: Average performance comparison of 100 times independent optimization

\begin{tabular}{llll}
\hline & Average optimum fitness /\% & Mean operation time /s & Average iteration time \\
\hline Anchorage aided location & 7.63 & 54.66 & 54.95 \\
algorithm & 8.11 & 53.87 & 54.12 \\
Free location algorithm & 9.89 & 34.73 & 33.71 \\
Algorithm in this paper & & & 3 \\
\hline
\end{tabular}

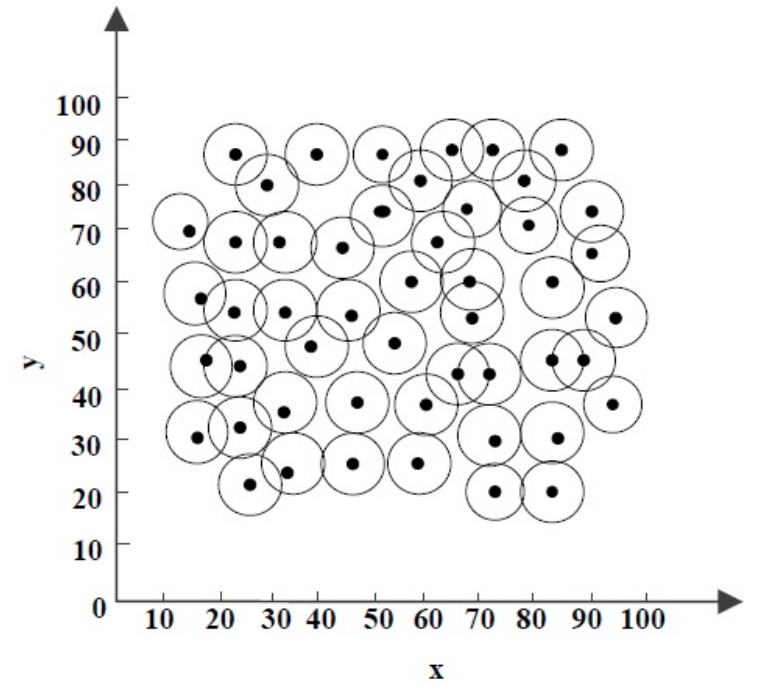

Figure 8: Node selection results of WSN algorithm in this paper

As shown in Figure 2, with the increase of beacon speed, the average root mean square error increases gradually, and the degree of increase gradually increases. When the moving beacon speed is below $2 \mathrm{~m} / \mathrm{s}$, the average root mean square error is basically stable at $0.6 \mathrm{~m}$. When the speed is $5 \mathrm{~m} / \mathrm{s}$, the average root mean square error is 8.34 $\mathrm{m}$. Although the moving time of the beacon is certain, the speed of the mobile beacon increases and the distance between the two continuous packets sent by the beacon increases, which leads to the density of the virtual beacons received by some unknown nodes. However, some unknown nodes receive sparse virtual beacons, leading to an increase in average root mean square error.

From Figure 3, we can see that the location coverage decreases with the increase of the moving speed of the beacon. When the speed is below $2 \mathrm{~m} / \mathrm{s}$, the coverage rate reaches $100 \%$. When the speed is $5 \mathrm{~m} / \mathrm{s}$, the coverage rate is only $71 \%$. Although the moving time of the mobile beacon is certain, the speed of the mobile beacon increases, the distance between the virtual beacons is increased, and the virtual beacons received by some unknown nodes are dense, so that enough location information can be obtained. And some unknown nodes receive virtual beacons sparsely, sometimes even cannot receive virtual beacons, which leads to the reduction of location coverage.

From Figure 4, we can see that with the gradual increase of the mobile beacon's moving time, the coverage of unknown nodes will gradually increase. When the beacon's moving time is $500 \mathrm{~s}$, the location coverage is only $69.6 \%$. When the moving time is more than $2500 \mathrm{~s}$, the location coverage is stable to $100 \%$.

As seen in Figure 5, with the increase of the number of EKF iterations, the initial location of the unknown nodes calculated by the weighted centroid method can be converged to the real position, so the average root mean square error will gradually decrease. When the number of iterations is 50 , the root mean square error is $5.1 \mathrm{~m}$. When the number of iterations is 350 , the mean root mean square error is basically stable at about $0.9 \mathrm{~m}$.

Through simulation and analysis, it is demonstrated that the proposed algorithm can achieve high locating accuracy in non-dense distribution, and the algorithm is relatively simple, without complex computation. In the same case, the locating effect is obviously superior to the anchor assistant location algorithm and the free localization algorithm. The locating error is mainly related to the moving time, moving speed, the number of virtual beacon points received and the number of iterations. The longer the beacon moves, the slower the speed is, the more the number of beacons is, the larger the number of iterations is, the smaller the root mean square error is and the higher the locating accuracy is. However, due to the low cost and low power consumption of the wireless sensor network, in practical application, the moving time of the beacon cannot be too long, the running speed cannot be too high or too low, the number of virtual beacons cannot be too great, and the number of iterations should not be too high in practical applications. The optimal location parameters should be chosen according to the locating accuracy tradeoff in the specific application. According to the simulation results, when the beacon's moving time is $3000 \mathrm{~s}$, the moving speed is $2 \mathrm{~m} / \mathrm{s}$, the number of virtual beacons is 21 and the number of iterations is 350 times, its locating effect is best, and it can meet the location requirements of the wireless sensor network in the general situation. 
As shown in Figures 6, 7 and 8, the coverage rate of wireless sensor networks is better under the optimal path selection algorithm and the coverage rate of the other two algorithms is more decentralized in the process of optimizing the node selection process for a non-dense distributed sensing network. The other two algorithms are used to optimize the selection of wireless sensor network nodes. The uniformity of node distribution is poor, and the coverage area of repetitive nodes is greater. The proposed algorithm is used to select wireless sensor network nodes, not only the node uniformity is higher than the other two algorithms, but also the coverage area of repetitive nodes is significantly reduced. It shows that the algorithm ensures the balance of network energy consumption and prolongs the network lifetime.

It is seen from Table 1 that in the average best fitness contrast, the best fitness of the proposed algorithm is $9.89 \%$, the anchor assistant location algorithm is $7.63 \%$, the free location algorithm is $8.11 \%$, and the algorithm has the highest fitness. In the comparison of average computing time, the algorithm used in this paper is $34.73 \mathrm{~s}$, compared to that of anchor assistant location algorithm (54.66 s) and the free location algorithm (53.87 s). The proposed algorithm has the shortest time and the highest efficiency. As summarized in Table 1 the proposed algorithm has the minimum number of iterations and the fastest convergence speed. The algorithm can quickly and effectively accomplish the node selection optimization of sensor networks in non- dense node distribution. The proposed algorithm not only has better dynamic node selection performance, but also has fast convergence speed and less computation time.

It can be seen that the proposed algorithm has the following advantages:

The fact that all nodes in traditional WSN are stationary is overcome. It only uses a GPS function, and can move the beacon to traverse the entire sensing area beforehand, reduces the number of actual stationary beacons that need to be arranged, thus greatly saving the location cost.

The Gauss Markov model is used to move the beacon. The parameters of the model, such as the moving time, the moving speed and the randomness adjustment parameters, are all adjustable, and the different parameters can be suitable for the location applications of wireless sensor networks with different sizes and precision requirements.

The unknown node passively receives the virtual beacon information in the process of location, and does not need the direct communication between the unknown node and the mobile beacon, thus greatly saving the communication cost and network cost.
The algorithm is scalable and the beacons can adopt different mobile models. If the number of mobile beacons is increased, the locating effect can be further improved, but the cost will be increased at the same time. Therefore, the suitable number of mobile beacons should be taken into consideration.

The algorithm is relatively simple and feasible, and the computational complexity is relatively low. The algorithm has high locating accuracy, robustness and fault tolerance. Occasionally, a single node failure does not affect the whole locating process.

It can enhance the clustering performance of the optimal nodes of the wireless sensor network, and has better performance of dynamic node selection in wireless sensor network, and the convergence speed is faster and the operation time is less.

\section{Conclusions}

In this paper, the optimal selection of mobile beacon path for sensor networks under non-dense distribution is designed to locate the mobile beacon nodes in the sensor network and to calculate the optimal location of the best energy node of the wireless sensor network, so as to obtain the optimal beacon path. This method can effectively solve the complexity and inefficiencycomputation of traditional algorithms, and can save communication costs and network costs, and has a good application value.

\section{References}

[1] Huang J.F., Chang G.Y., Chen G.H., A Historical-Beacon-Aided Localization Algorithm for Mobile Sensor Networks, IEEE Trans. Mobile Comput., 2015, 14(6), 1109-1122.

[2] Halder S., Ghosal A., A Survey on Mobile Anchor Assisted Localization Techniques in Wireless Sensor Networks, Wireless Networks, 2015, 22(7), 1-20.

[3] Alomari A., Comeau F., Phillips W., New Path Planning Model for Mobile Anchor-Assisted Localization in Wireless Sensor Networks, Wireless Networks, 2017, (8), 1-19.

[4] Sun D., Leung V.C.M., Qian Z. Beacon Deployment Strategy for Guaranteed Localization in Wireless Sensor Networks, Wireless Networks, 2016, 22(6), 1947-1959.

[5] Singh M., Khilar P.M., An Analytical Geometric Range Free Localization Scheme Based on Mobile Beacon Points in Wireless Sensor Network, Wireless Networks, 2015, 22(8), 1-14.

[6] Batra P.K., Kant K., LEACH-MAC: A New Cluster Head selection algorithm for Wireless Sensor Networks, Wireless Networks, 2016, 22(1), 49-60.

[7] Say S., Ernawan M.E., Shimamoto S., Cooperative Path Selection Framework for Effective Data Gathering in UAV-Aided Wire- 
less Sensor Networks, Leice . Comm., 2016, 99(10), 2156-2167.

[8] Zhou Y., Lin T., Zeng G., Label Free Detection of Lead Using Impedimetric Sensor Based on Ordered Mesoporous Carbon-Gold Nanoparticles and Dnazyme Catalytic Beacons, Talanta, 2016, 146, 641-647.

[9] Pilania V., Gupta K., Localization Aware Sampling and Connection Strategies for Incremental Motion Planning Under Uncertainty, Autonom. Robots, 2017, 41(1), 1-22.

[10] Sun A.L., Zhang Y.F., Wang X.N., Sensitive Voltammetric Determination of Dna Via a Target-Induced Strand-Displacement Reaction Using Quantum Dot-Labeled Probe DNA, Microchimica Acta, 2015, 182(7-8), 1403-1410.

[11] Güler S., Fidan B., Dasgupta S., Adaptive Source Localization Based Station Keeping of Autonomous Vehicles, IEEE Trans. Autom. Contr., 2017, 62(7), 3122-3135.

[12] Wang M., Hou X., Wiraja C., Smart Magnetic Nanosensors Synthesized through Layer-by-Layer Deposition of Molecular Beacons for Noninvasive and Longitudinal Monitoring of Cellular mRNA, Acs Appl. Mat. Interfaces, 2016, 8(9), 5877.

[13] Green J., Pérez-Olivas H., Martínez-Díaz S., VLC-beacon Detection with an Under-Sampled Ambient Light Sensor, Optics Comm., 2017, 397, 122-128.

[14] Rao P. C. S., Banka H., Energy Efficient Clustering Algorithms for Wireless Sensor Networks: Novel Chemical Reaction Optimization Approach, Wireless Networks, 2015, 23, 1-20.

[15] Kumar R., Kumar D., Kumar D., EACO and FABC to Multi-Path Data Transmission in Wireless Sensor Networks, IET Comm., 2017, 11(4), 522-530.

[16] Cai W., Zhang M., 3D Dubins Curves Based Path Programming for Mobile Sink in Underwater Sensor Networks, Electr. Lett., 2016, 53(1), 48-50.

[17] Ahn N., Park S., An Optimization Algorithm for The Minimum K -Connected M -Dominating Set Problem in Wireless Sensor Networks, Wireless Networks, 2015, 21(3), 783-792.

[18] Rao P.C.S., Jana P.K., Banka H., A Particle Swarm Optimization Based Energy Efficient Cluster Head Selection Algorithm for Wireless Sensor Networks, Wireless Networks, 2017, 23(7), 2005-2020.

[19] Sandhya M.K., Murugan K., Devaraj P., Selection of Aggregator Nodes and Elimination of False Data in Wireless Sensor Networks, Wireless Networks, 2015, 21(4), 1327-1341.
[20] Azharuddin M., Jana P.K., A Distributed Algorithm for Energy Efficient and Fault Tolerant Routing in Wireless Sensor Networks, Wireless Networks, 2015, 21(1), 251-267.

[21] Hu Y.F., Ding Y.S., Ren L.H., An Endocrine Cooperative Particle Swarm Optimization Algorithm for Routing Recovery Problem of Wireless Sensor Networks With Multiple Mobile Sinks, Informat. Sci., 2015, 300, 100-113.

[22] Alia O.M., Dynamic Relocation of Mobile Base Station in Wireless Sensor Networks Using a Cluster-Based Harmony Search Algorithm, Informat. Sci., 2017, S385-386, 76-95.

[23] Zhang P., Hongjuan C., Research on Safety Monitoring in Active Sensor in Power System, Automat. Instrument., 2017, 11(4), 1317-1121.

[24] Qin Z.C., Zhou C.H., Zhang P., Energy Efficient Sensing Strategy for Cognitive Radio Sensor Networks, J. China Acad. Electr. Inform. Technol., 2015, 23(3), 313-792.

[25] Zhang P., Zhang Q., Song S., Permanent Magnet Motor Vector Control Using Hall Effect Position Sensor, J. Power Supply, 2017, 14(3), 453-254.

[26] Fu H., Liu X., A Study on the Impact of Environmental Education On Individuals' Behaviors Concerning Recycled Water Reuse, Eurasia J. Math. Sci. Technol. Educat., 2017, 13(10), 6715-6724.

[27] Attia G.F., Abdelaziz A.M., Hassan I.N., Video Observation of Perseids Meteor Shower 2016 from Egypt. Appl. Math. Nonlin. Sci., 2017, 2(1), 151-156.

[28] Kim N.Y., Jeon E.J., Jung S.H., Ahn S.J., Park M.A., Seo J.S. Gene Expression Profiling and Expression Analysis of Freshwater Shrimp (Neocaridina Denticulata Denticulata) Using Expressed Sequence Tags and Short-Term Exposure to Copper. J. Envir. Biol., 2018, 39(1), 51-57.

[29] Gao W., Wang W.F., The Fifth Geometric-Arithmetic Index of Bridge Graph and Carbon Nanocones, J. Diff. Eq. Appl., 2017, 23(1-2SI), 100-109.

[30] Khellat F., Khormizi M.B., A Global Solution for a ReactionDiffusion Equation on Bounded Domains, Appl. Math.Nonlin. Sci., 2018, 3(1), 15-22.

[31] Guadalupe Sanchez-Duarte R., Del Rosario Martinez-Macias M., Araceli Correa-Murrieta M., Saldivar-Cabrales J., Isabel Sanchez-Machado D., Lopez-Cervantes J., Synthesis of Chitosan Hydrogels From Shrimp Shell for Copper Adsorption Tests, Rev. Int. Contamin. Ambient., 2017, 33(SI), 93-98. 